\title{
Mitochondrial ultrastructure and markers of dynamics in hepatocytes from aged, calorie restricted mice fed with different dietary fats
}

\author{
Husam Khraiwesh ${ }^{\mathrm{a}}$, José A. López-Domínguez ${ }^{\mathrm{a}, \mathrm{b}}$, Lucía Fernández del Río ${ }^{\mathrm{a}}$, Elena \\ Gutierrez-Casado $^{a}$, Guillermo López-Lluch ${ }^{c}$, Plácido Navas ${ }^{c}$, Rafael de Cabo ${ }^{d}$, Jon J. \\ Ramsey $^{b}$, María I. Burón ${ }^{a}$, José M. Villalba ${ }^{a},{ }^{,}$, and José A. González-Reyes ${ }^{a}{ }^{, *}$ \\ aDepartamento de Biología Celular, Fisiología e Inmunología, Universidad de Córdoba, Campus \\ de Excelencia Internacional Agroalimentario, ceiA3, Córdoba, Spain \\ bVM Molecular Biosciences, University of California, Davis, United States \\ 'Centro Andaluz de Biologia del Desarrollo, Universidad Pablo de Olavide-CSIC, CIBERER, \\ Instituto de Salud Carlos III, Sevilla, Spain \\ dLaboratory of Experimental Gerontology, National Institute of Aging, National Institutes of Health, \\ Baltimore, MD, United States
}

\begin{abstract}
In this paper we analyzed changes in hepatocyte mitochondrial mass and ultrastructure as well as in mitochondrial markers of fission/fusion and biogenesis in mice subjected to $40 \%$ calorie restriction (CR) for 18 months versus ad libitum-fed controls. Animals subjected to CR were separated into three groups with different dietary fats: soybean oil (also in controls), fish oil and lard. Therefore, the effect of the dietary fat under CR was studied as well. Our results show that $\mathrm{CR}$ induced changes in hepatocyte and mitochondrial size, in the volume fraction occupied by mitochondria, and in the number of mitochondria per hepatocyte. Also, mean number of mitochondrial cristae and lengths were significantly higher in all CR groups compared with controls. Finally, CR had no remarkable effects on the expression levels of fission and fusion protein markers. However, considerable differences in many of these parameters were found when comparing the $\mathrm{CR}$ groups, supporting the idea that dietary fat plays a relevant role in the modulation of CR effects in aged mice.
\end{abstract}

\section{Keywords}

Calorie restriction; Dietary fat; Hepatocyte; Mitochondria; Mature/old-aged mice

\footnotetext{
(C) 2014 Elsevier Inc. All rights reserved.

*Corresponding author. ${ }^{* *}$ Correspondence to: J.A. González-Reyes, Departamento de Biología Celular, Fisiología e Inmunología, Universidad de Córdoba, Campus de Rabanales, Edificio Severo Ochoa, $3^{\mathrm{a}}$ planta, Campus de Excelencia Internacional Agroalimentario, ceiA3, 14014 Córdoba, Spain. Tel.: +34 957218595; fax: +34 957218634. bc1gorej@uco.es (J.A. González-Reyes). Conflict of interest

The authors declare no conflicts of interests.
} 


\section{Introduction}

Aging, a nearly universal feature of biological organisms, has been defined as a timedependent degenerative process caused by accumulated damage that leads to cellular dysfunction, tissue failures, and death (Bratic and Larsson, 2013; Campisi, 2013). Although the mechanisms underlying aging are still unknown, several hallmarks have been proposed to explain the molecular and physiological basis of aging (López-Otín et al., 2013). Some of these hallmarks point out the mitochondria to play an essential role in the aging process.

Although aging has been traditionally explained on the basis of the action of free radicals, especially those generated in and acting on the mitochondria (Harman, 1972), the so-called "mitochondrial free radical theory of aging" (Miquel et al., 1980) has been challenged (see for example, Hekimi et al., 2011). However, excessive reactive oxygen species (ROS) production and accumulation are still considered to be involved in the development of different pathologies and the aging process (see Barja, 2013 for a recent review). On the other hand, additional alternative pathways, such as deregulated nutrient sensing, have been recently proposed to explain the effect of aging on mitochondrial function. (Chung et al., 2013; López-Otín et al., 2013).

Calorie restriction (CR; i.e. a reduction in calorie intake without malnutrition) is a powerful tool for investigating aging (Colman et al., 2009; Sohal and Weindruch, 1996; Weindruch and Walford, 1988), without the need for genetic manipulation or pharmacologic treatments. Thus, the reduction in calorie intake (typically $20-40 \%$ of the ad libitum fed controls) has been repeatedly reported to increase life span and to prevent cancer, diabetes, hypertension and other age-related diseases in a wide range of animals, including nonhuman primates and humans (Colman et al., 2009; Mattison et al., 2012; Weindruch and Sohal, 1997). Although the mechanisms by which CR operates are not completely understood, it is often assumed that the anti-aging action of $\mathrm{CR}$ is based, at least partially, on its ability to suppress oxidative stress and maintain the cellular redox status to provide optimal cell signaling properties and normal gene expression (Chung et al., 2013).

Decreased damage to proteins and DNA (especially mitochondrial DNA) has been reported in CR animals (Kaneko et al., 1997; Pamplona et al., 2002; Sohal et al., 1994; Youngman et al., 1992). Another target of ROS are membrane phospholipids, especially those containing double bounds in their hydrophobic tails, and an inverse correlation between life span and the degree of membrane phospholipid unsaturation has been proposed (Hulbert, 2003; Pamplona et al., 2002). The assumption has been made that polyunsaturated fatty acids are more susceptible to peroxidation and other modifications that result in the accumulation of oxidative injury in membranes containing these fatty acids. This idea is supported by a decreased content of long-chain polyunsaturated fatty acids in mitochondria isolated from different organs after CR (Yu et al., 2002). As one of the major ROS-producing constituents of the cell, special attention has been paid to the role of the mitochondria on these phenomena, and the proposal has been made that a combination of both "mitochondrial" and "membrane" theories of aging (Zs.-Nagy, 1978) can explain the life-span extension effect of CR (Bevilacqua et al., 2004; Hagopian et al., 2005). 
Different experiments have been carried out feeding animal diets in which the composition of the dietary fat was controlled in an attempt to elucidate the precise role of membrane lipid composition on CR effects. In this sense, we reported changes in lipid composition and some physiological parameters of liver and skeletal muscle mitochondria isolated from mice fed for 1 month with $40 \%$ CR diets with different dietary fat sources (soybean oil, fish oil, and lard) compared with controls fed ad libitum (Chen et al., 2012, 2013). More recently, following an identical feeding protocol we found a significant decrease in skeletal muscle apoptosis in mice following 6 months of CR, and this phenomenon was modulated by dietary fat since animals fed with a CR diet containing fish oil showed an enhanced protection against apoptosis. We hypothesized that this phenomenon could be mechanistically linked to a protective action of CR against sarcopenia with aging (LópezDomínguez et al., 2013).

Due to its role as the primary organ for fat, carbohydrate, and protein interconversion between storage and metabolizable forms, as well as for its multiplicity of functions, liver has been extensively used as a model to study the effects of CR and aging. Thus, LópezLluch et al. (2006) reported an increase in mitochondrial mass in rats subjected to $40 \% \mathrm{CR}$ through enhanced mechanisms of mitochondrial biogenesis. More recently, we have shown changes in hepatocytes from young mice subjected to 6 months of $40 \%$ calorie restriction (Khraiwesh et al., 2013). Under these conditions, we have reported significant increases in both cell volume fractions occupied by mitochondria as well as in mean number of mitochondria per hepatocyte. These changes were accompanied by increased number of mitochondrial cristae in CR fed animals. Also, we detected changes in the expression levels of proteins related to mitochondrial fission (such as Fis1 and Drp1) and fusion (OPA1, Mfn1 and Mfn2). Moreover, in that paper we reported also that different dietary fats in CR animals can modulate mitochondrial ultrastructure and fission/fusion marker dynamics, highlighting the possible role of dietary fat in mitochondrial function of hepatocytes from CR animals (Khraiwesh et al., 2013). In accordance, dietary fat also modulated apoptosis in aging liver (López-Domínguez et al., in press).

In this paper we studied the effects of 18 months of CR in mature/old mice(21 months old)on basic morphological and fission/fusion parameters of hepatocyte mitochondria versus their ad libitum (AL) fed controls as well as the effects of different dietary fats (lard, soybean oil and fish oil) in CR animals. Furthermore, we have also studied protein expression levels of the master regulator of mitochondrial biogenesis, peroxisome proliferator activated receptor gamma co-activator 1 alpha (PGC-1a) and one of its major target, the nuclear respiratory factor 1 (Nrf1). Finally, we also determined lipid peroxidation levels in liver cell homogenates isolated from the above-mentioned animals. Our results showed that, in general, CR increased stereological parameters of mitochondria and induced changes in mitochondrial ultrastructure and fission/fusion markers as well as in PGC-1a and in Nrf1 protein expression and lipid peroxidation levels. However, some considerable differences were found when comparing the different CR groups, indicating amodulatory effect of the dietary fat in regulating mitochondrial mass in mature/old mice maintained on long-term $\mathrm{CR}$. 


\section{Material and methods}

\subsection{Animals and diets}

Male C57BL/6 mice (Charles River Laboratories, Spain) were bred and raised in a vivarium at the Centro Andaluz de Biología del Desarrollo (CABD) under a $12 \mathrm{~h}$ light/dark cycle (8:00 am-8:00 pm), at $22 \pm 3{ }^{\circ} \mathrm{C}$ and under controlled humidity. The animals were fed with a commercial rodent chow diet (Harlan Teklad \#7012, Madison, WI) until they were 3 months old. The mice were then randomly assigned into 4 dietary groups and fed with a modified AIN-93G semi-purified diet containing $20.3 \%$ protein, $63.9 \%$ carbohydrate, and $15.8 \%$ fat (\% total $\mathrm{Kcal} / \mathrm{d}$ ). In order to prevent obesity during the study, the control group was fed $95 \%$ of a pre-determined ad libitum intake (12.5 Kcal). CR dietary groups were fed $40 \%$ less calories, these diets being identical except for dietary lipid source, which was soybean oil (high in n-6 polyunsaturated fatty acids, PUFAs, Super Store Industries, Lathrop, CA) for the ad libitum fed mice and one of the CR groups. The two remaining CR groups were fed with diets containing fish oil (high in n-3 PUFAs: 18\% EPA, 12\% DHA, Jedwards International, Inc. Quincy, MA), or lard (high in saturated and monounsaturated fatty acids, ConAgra Foods, Omaha, NE). To ensure adequate linoleic acid levels, the CRFish diet also contained soybean oil (14\% of total fat content). The main properties of fatty acids present in the three dietary fats are shown in Table 1. For a detailed description of fatty acid compositions of all diets used in this paper see our previous publications (Chen et al., 2012, 2013). All mice were housed individually with free access to water. Food was replaced every day between 8:00 and 9:00 am.

After a dietary intervention period of 18 months, animals were sacrificed by cervical dislocation following an 18 hour fast and quickly dissected to obtain liver samples that were used for ultrastructural analysis, as well as for homogenates, cytosolic and mitochondriaenriched fractions isolated for hydroperoxide determinations and studies of mitochondrial dynamics. All experimental procedures and animal handling were in accordance with the Pablo de Olavide University Ethical Committee rules, and the 86/609/EEC Directive on the protection of animals used for experimental and other scientific purposes.

\subsection{Tissue processing for microscopy}

The organs were removed and quickly washed, and then they were cut in small pieces of about $1 \mathrm{~mm}^{3}$. Samples were fixed, dehydrated and embedded in EMbed 812 epoxy resin as previously described (Khraiwesh et al., 2013). Blocks were sectioned in an Ultracut Reichert ultramicrotome to get both semithick $(0.5-1 \mu \mathrm{m}$ width) and ultrathin $(40-60 \mathrm{~nm}$ width) sections for different purposes as described in the following paragraphs. For each animal, we processed and examined about five to six liver pieces taken from the different lobules of the organ. About five to seven animals were processed and analyzed in every dietary group. We mounted and observed a minimum of two grids taken from different parts of each block.

\subsection{Hepatocyte size}

Semithick sections were mounted on glass slides and stained with $1 \%$ toluidine blue in a $1 \%$ borax aqueous solution for $2 \mathrm{~min}$. Micrographs were obtained using a Leica DME light microscope and planimetric parameters of whole hepatocytes were measured using ImageJ 
software (N.I.H.). Cells were selected on the basis of showing a central nucleus and a circularity coefficient ranging from 0.85 to 1.0 . About $1100-1200$ hepatocytes from five animals per diet were measured. Final volumes were calculated assimilating hepatocyte shape to spheroids.

\subsection{Mitochondrial ultrastructure and stereology}

Ultrathin sections were mounted on nickel grids. After staining in aqueous $4 \%$ uranyl acetate and modified Sato's lead citrate (Sato, 1968), the sections were viewed and photographed in a Philips CM-10 electron microscope. From these sections, we obtained low-magnification micrographs (originally $5800 \times$ ) for evaluating cell size as well as the number of mitochondria per cell section. Higher magnification pictures of mitochondria (originally $25,000 \times$ ) were used to obtain ultrastructural parameters (area, major and minor diameters, and circularity coefficient). These pictures were taken randomly from 35 to 40 hepatocytes per experimental group. We also measured crista length on these pictures using planimetric techniques. In these cases, mitochondria were selected on the basis of showing a well-defined cristal ultrastructure.

Stereological analysis of the samples was carried out in order to get information on mitochondrial volumetric parameters. Volume density (Vv) and numerical density (Nv) values were obtained following a point-counting method analysis according to Weibel (1979). We used a simple square-lattice test system of $5 \mathrm{~mm}$ spacing (equivalent to $0.4 \mu \mathrm{m}$ ), which was applied to obtain $\mathrm{Vv}$ of mitochondria. The numerical density of mitochondria (Nv) was obtained using the formula

$$
\mathrm{N}_{\mathrm{v}}=\frac{\mathrm{KNa}^{3 / 2}}{\beta \mathrm{Vv}^{1 / 2}}
$$

where "Na" represents the number of mitochondria per $\mu \mathrm{m}^{2}$ of cell and $\mathrm{k}$ and $\beta$ the mitochondrial size distribution and shape coefficient, respectively. These coefficients were calculated using the results of planimetric measurements and assimilating mitochondrial shape to regular prolate spheroids (Weibel, 1979). Planimetric measurements on mitochondria were performed using ImageJ software (N.I.H.).

\subsection{Total mitochondrial mass and number of mitochondria per cell}

These parameters were calculated by multiplying mean $\mathrm{Vv}$ and $\mathrm{Nv}$ by mean hepatocyte volume, respectively. For comparisons, standard deviations were accumulated.

\subsection{Preparation of homogenates, cytosolic and mitochondria-enriched fractions from liver}

Livers, without fat or connective tissue, were minced and homogenized at $4{ }^{\circ} \mathrm{C}$ in ice-cold isolation buffer containing $5 \mathrm{mM}$ Tris- $\mathrm{HCl} \mathrm{pH}$ 7.4, $0.225 \mathrm{M}$ mannitol, $0.075 \mathrm{M}$ sucrose, 0.5 $\mathrm{mM}$ ethylene glycol tetraacetic acid, $1 \mathrm{mM}$ phenylmethylsulfonyl fluoride, and $20 \mu \mathrm{g} / \mu \mathrm{l}$ each chymostatin, leupeptin, antipain, and pepstatin with the aid of a Teflon-glass issue homogenizer. An aliquot from the total homogenate was re-homogenized using a mechanical tissue disrupter (Ultra-Turrax T25,IKA; Staufen, Germany) during $30 \mathrm{~s}$ and then 
stored at $-80{ }^{\circ} \mathrm{C}$ until further analysis. The remaining homogenate was further processed for isolation of mitochondria-enriched fractions and cytosols. Briefly, samples were centrifuged at $600 \mathrm{~g}$ for $5 \mathrm{~min}$ and the pellet was discarded. Additional centrifugation steps were performed until no pellet at $600 \mathrm{~g}$ was observed. The final supernatant was then centrifuged for $15 \mathrm{~min}$ at $6500 \mathrm{~g}$ to obtain a mitochondrial-enriched fraction. Mitochondria were resuspended in $200 \mu \mathrm{l}$ of isolation buffer and stored frozen at $-80{ }^{\circ} \mathrm{C}$ until further analysis. Supernatants were centrifuged again at 80,000 $\mathrm{g}$ for $45 \mathrm{~min}$ to separate a cytosolic fraction.

\subsection{Polyacrylamide gel electrophoresis and western blot immunodetection of mitochondrial fission- and fusion-related proteins}

Protein $(50 \mu \mathrm{g})$ was diluted in loading buffer $(60 \mathrm{mM}$ Tris- $\mathrm{HCl} \mathrm{pH} 6.8,10 \%$ sucrose, $2 \mathrm{mM}$ ethylenediaminetetraacetic acid, 1.5\% (w/v) SDS, $20 \mathrm{mM}$ dithiothreitol, $0.01 \%$ (w/v) bromophenol blue) and denatured by heating prior to separation by electrophoresis in 4$20 \%$ polyacrylamide gradient gels. We have used homogenate to analyze PGC-1a and Nrf1 (nuclear respiratory factor 1) and hydroperoxide levels were determined in homogenates; mitochondrial fraction to immunodetect Mfn1, Mfn2, Fis1, Drp1 and Opa1; and cytosolic fraction to measure Drp1. Proteins were then blotted onto nitrocellulose sheets and Ponceau $\mathrm{S}$ staining was used to visualize protein lanes. Membranes were scanned in a GS-800 densitometer (Bio-Rad) and quantified using Quantity One software (Bio Rad), as an internal control of actual protein load. Data normalization using Ponceau S staining was previously validated by our group (Bello et al., 2003). Primary polyclonal antibodies against Opa1 (goat polyclonal antibody), Fis1, Drp1, Mfn1, Mfn2, PGC-1a and Nrf1 (rabbit polyclonal antibody) were obtained from Santa Cruz Biotech and diluted at a range of 1:1000-1:3000. Secondary anti-IgG antibodies (Sigma) with horseradish peroxidase activity and enhanced chemiluminescence (ECL-Plus, GE Healthcare Life Sciences) were used to detect binding sites. CR-Soy and control diets were compared in a first set of electrophoresis gels and blots, and differences between CR diets (CR-Lard, CR-Soy and CR-Fish) were assessed in an additional set of gels, to allow analysis of a higher number of samples for each case. Consequently, protein levels are represented in separate graphs.

\subsection{Measurement of lipid hydroperoxides}

Lipid hydroperoxide levels were determined in liver homogenate fractions (100 $\mu \mathrm{g}$ protein) according to the method of Jiang et al. (1991), based on the measurement of hydroperoxidemediated oxidation of $\mathrm{Fe}^{2+}$ to $\mathrm{Fe}^{3+}$ in the presence of xylenol orange under acidic conditions. Hydroperoxides were determined by direct incubation of membranes with xylenol orange reagent for $75 \mathrm{~min}$ at room temperature in the dark. Absorbance of $\mathrm{Fe}^{3+}$ xylenol orange complex was recorded at $560 \mathrm{~nm}$ (extinction coefficient $43 \mathrm{mM}^{-1} \mathrm{~cm}^{-1}$ ). Linearity of reaction was tested by constructing a standard plot with tert-butyl hydroperoxide. We performed these determinations in samples from animals fed with experimental diets for 6 and 18 months. 


\subsection{Statistic analysis}

Values were expressed asmean \pm SEM. D'Agostino-Pearson tests were performed to determine data normality. The effect of CR was assessed by Student's $t$ test (CR-Soy group vs AL-Soy group). In case the data did not pass the normality test, the nonparametric MannWhitney test was followed. The effect of dietary fat under CR was assessed by one-way ANOVA followed by a post-hoc analysis (Tukey's test for multiple comparisons) to assess for significant differences among groups. Post-hoc analysis of linear trend was also performed to investigate putative alterations of tested parameters among CR diets ordered as CR-Lard $\rightarrow$ CR-Soy $\rightarrow$ CR-Fish, which resulted in a progressive increase of the $n-6 / n-3$ ratio in phospholipid HUFA (Table 1, see also Chen et al., 2012). In case the data did not pass the normality test, the nonparametric Kruskal-Wallis test was followed. Means were considered statistically different with $\mathrm{p}<0.05$. All statistical analyses were performed using Graphpad Prism 5.03 (Graphpad Software Inc., San Diego, CA, USA).

\section{Results}

\subsection{Hepatocyte size}

When examined under the electron microscope, hepatocyte morphology was similar in all four experimental groups and fits well with classical description in the literature of these cells in old mice (see for example, Pieri et al., 1980; Schmucker, 1990; Schmucker et al., 1978). In general, a high proportion of the cell was occupied by a central and well developed nucleus, and binucleated cells were frequently observed. The surrounding cytoplasm contained a high number of mitochondria (see Fig. 1A - D). At higher magnification, mitochondria displayed a typical ellipsoidal morphology with most of the cristae perpendicular to the longer axis of the organelle (Fig. 2A - D). No striking morphological differences were found in mitochondrial ultrastructure by examination of the pictures with the naked eye. However, significant changes were found when accurate quantitative analyses were applied (see below).

The planimetric measurement performed on whole hepatocytes using semithick sections yielded the results displayed in Table 2. Increased cell size was found in those animals fed with diets enriched in fish or soybean oils under calorie restriction (i.e. CR-Soy and CRFish) in comparison to the AL group. However, a significant decrease in this parameter was found in animals fed with a CR diet enriched in lard (CR-Lard group) compared to the other CR groups. These results affected all the parameters used to evaluate cellular size (major and minor diameters as well as cell area and volume). Cell shape also changed in the different groups (Table 2), and those hepatocytes showing the smallest sizes (CR-Lard) displayed more spherical shape, and animals from the CR-Soy showed more elliptical shape although this was only significantly different from the CR-Lard group (see Table 2).

\subsection{Ultrastructural analysis of mitochondria and cristae}

The results of planimetric analysis performed on individual mitochondria from AL and CR fed animals are summarized in Table 3. We found that CR induced a significant increase in both minor and major mitochondrial diameters regardless of the fat source and concomitant increases in mitochondrial section area and mitochondrial volume. However, some changes 
were detected when comparing the different $\mathrm{CR}$ groups. In particular, mice from the CRLard and CR-Soy groups exhibited larger mitochondria than those from the CR-Fish group.

The number of cristae per mitochondrion, as well as their lengths, was scored and the results are shown in Table 4. A significant increase in the mean number of cristae per mitochondrion was observed in all of the CR animals compared to those fed ad libitum, but no differences were found among the CR groups. Mean crista length was significantly decreased in the AL group compared to the CR groups. Mean crista length also differed significantly among all of the CR groups with CR-Soy > CR-Lard > CR-Fish.

3.2.1. Stereological analysis of hepatocyte mitochondria-In addition to the planimetric measurements on mitochondria, we performed a stereological analysis in order to obtain information about possible changes in the cell volume fraction occupied by mitochondria (volume density, $\mathrm{Vv}$ ) and the number of mitochondria perhepatocyte volume unit (numerical density, Nv) and the results are displayed in Figs. 3 and 4, respectively. In general, mitochondria occupied $10 \%$ to $16 \%$ of hepatocyte volume. All CR groups showed a significant increase in Vv compared with AL-fed animals, whereas no differences among the three CR groups were observed. Numerical density also increased in animals under CR regardless of the dietary fat source. However, differences among the $\mathrm{CR}$ groups were observed, with the CR-Soy showing significantly decreased Nv when compared to the CRFish group (see Fig. 4).

\subsection{Mitochondrial mass and number of mitochondria per hepatocyte}

Using the data from cellular volumes summarized in Table 2 and $\mathrm{Vv}$ and NV shown in Figs. 3 and 4 , it is possible to calculate the actual cell volume occupied by mitochondria (mitochondrial mass) as well as the mean number of mitochondria per cell. These data, obtained by multiplying each mean hepatocyte volume by its mean Vv or Nv, respectively, are shown in Table 5. Mitochondrial mass and number increased under CR condition, and significant differences were also observed when comparing the different CR groups. Among the three CR groups, the lowest cell volume occupied by mitochondria was observed in CRLard compared with either the CR-Soy or CR-Fish groups; whereas the highest number of mitochondria per cell was found in the CR-Fish compared to either the CR-Lard or CR-Soy groups.

\subsection{Analysis of mitochondrial fission and fusion markers}

In addition to the structural and ultrastructural studies, we also performed an analysis on the expression of proteins related to mitochondrial fission and fusion. Thus, Fis1, Mfn1, Mfn2, and OPA1, which are mitochondrial intrinsic proteins, were determined in mitochondriaenriched fractions while Drp1, which resides mostly in the cytosol being recruited to mitochondria to promote fission events, was determined in both cytosolic and mitochondria enriched fractions.

Fig. 5 shows the levels of fission proteins Drp1 and Fis1. In cytosolic fractions decreased values of Drp1 were found in CR animals compared to the AL fed controls (Fig. 5A), but no significant differences were detected when comparing the different CR groups (Fig. 5B). 
However, the expression level of Drp1 determined in mitochondria-enriched fractions remained unaltered when comparing $\mathrm{AL}$ and CR-Soy groups, and in animals fed with $\mathrm{CR}$ diets of different fat composition (Fig. 5C and D). In the case of Fis1, results were similar to those found for Drp1 in cytosolic fractions, since CR induced a decrease in its expression level, but no changes were detected among the CR groups (Fig. 5E and F).

On the other hand, fusion protein levels exhibited few changes in the different experimental groups. Thus, neither Mfn1 norMnf2 expression levels showed significant changes when comparing AL versus $\mathrm{CR}$ fed animals or when comparing the three CR groups (Fig. $6 \mathrm{~A}-$ D). Opa1 expression levels were also similar in AL compared to CR-soy group (see Fig. 6E), but a significant increase in this marker was found in animals fed with a CR diet containing soybean oil compared to the CR-Lard and CR-Fish groups (see Fig. 6F).

\subsection{Analysis of PGC-1a and Nrf1 protein expression}

The protein expression levels of PGC-1a were determined in mice after 6 and 18 months of $\mathrm{CR}$ as well as in control ad libitum fed mice, and the results are displayed in Fig. 7. In AL animals we found a significant increase during aging (Fig. 7A) but no changes were induced by $\mathrm{CR}$ in either young or mature/old mice. In both cases, these levels remained similar to those found in young control specimens (Fig. 7A). However, we found a significant increase in PGC-1a during aging in mice fed with diets containing lard as fat source (Fig. 7B). Similarly to CR-Soy group, we did not find changes in levels during aging in CR-Fish fed mice (Fig. 7B). The expression level of Nrf1 protein in the different groups and ages showed similar results to those displayed for PGC-1a. Thus, we found an increase during aging in AL mice but no changes in mice fed with CR-Soy diet (Fig. 8A). However, under CR feeding conditions fish oil and lard had significant impact on this factor since both fats induced increases in Nrf1 levels when comparing old/mature to young mice (Fig. 8B).

\subsection{Hydroperoxide content}

Hydroperoxide levels were analyzed in liver homogenates from mice after 6 and 18 months of CR and the results are shown in Fig. 9. No differences were found in young versus old/ mature mice fed with the AL diet, while CR induced significant changes depending on both age and dietary fat. Thus, aging increased significantly the levels of hydroperoxides in CRLard group, while no age-related changes were observed in CR-Soy fed animals. However, in CR-Fish fed mice, hydroperoxide levels significantly decreased after 18 months of CR when compared to their young counterparts (Fig. 9). Post hoc analysis of linear trend among CR diets ordered as CR-Lard, CR-Soy and CR-Fish was statistically significant in young animals, whereas no trend was found in the old groups (see Fig. 9).

\section{Discussion}

\subsection{Aging and calorie restriction induce changes in hepatocyte size}

Due to its multiple functions in the regulation of carbohydrate, lipid and protein metabolism, as well as its role in detoxification processes, the liver has been the target organ for many studies related to aging. Moreover, liver plays a pivotal role in coordinating metabolism in response to nutrition so that many of the benefits of calorie restriction (CR) or other 
nutritional interventions are probably mediated by direct effects of diet on hepatic function (Le Couteur et al., 2010). Since many of these effects are mediated by the hepatocytes, we have focused our work in this cell type and more specifically on the possible changes of mitochondria in mice subjected to long-term CR and fed with different dietary fats.

Although the liver seems to age fairly well in comparison with other organs (Schmucker, 1998; Schmucker and Sanchez, 2011), its structure undergoes a series of time-related changes (inflammation, pseudocapillarization, etc.) that have implications for many diseases (Cogger et al., 2013; Gregg et al., 2012; Le Couteur et al., 2010). However, in this work we have only considered hepatocytes showing a typical morphology similar to that described in the literature (see for example, Pieri et al., 1980; Schmucker, 1990) and possible modifications on cell types other than hepatocytes have not been analyzed. On the other hand, we have recently reported morphological changes in hepatocyte and mitochondria ultrastructure in young animals following feeding protocols identical to those described in the present study (see Khraiwesh et al., 2013). Therefore, an important part of this discussion will be focused on comparisons with these previous results in terms of aging. A brief summary of the differences found in young mice subjected to 6 months of CR and the results reported in this study is included in Table 6 .

The first difference we found in hepatocytes from animals subjected to CR for 18 months concerned cell size and substantial differences were also detected depending on the dietary fat. Thus, hepatocyte size increased in CR-Soy and CR-Fish groups while a significant decrease was found in mice fed with a CR-Lard diet. In young animals subjected for six month to CR, we observed increases in cell size in the groups consuming high levels of dietary polyunsaturated fatty acids (CR-Soy and CR-Fish), while no changes were detected in animals consuming high levels of monounsaturated or saturated fatty acids (CR-Lard) when compared to the AL controls (Table 6; Khraiwesh et al., 2013). Therefore, it is possible to assume that aging affected hepatocyte size in different ways depending on dietary fat composition. It is generally accepted that in rodents hepatocyte size increases during development and maturation and declines during senescence such that hepatocyte size becomes equivalent in volume in senescent and very young animals (Schmucker, 2005). In our case, we found cell size increases in all of the evaluated diets except for the CR-Lard fed animals, suggesting different mechanisms of cell adaptation to the aging process depending on the composition of dietary fat. According to these results, and following an identical experimental procedure, we have recently reported increases of nuclear area in animals following 18 month CR compared to their respective 6 month counterparts except for the CR-Lard group, in which nuclear sizes remained unaltered (López-Domínguez et al., in press).

\subsection{Mitochondrial ultrastructure}

In addition to hepatocyte size, aging and long-term CR induce other ultrastructural changes in hepatocyte organelles. Increases in polyploid and binucleate cells as well as in dense bodies and lipofuscin deposits and decreases in smooth endoplasmic reticulum surface have been frequently reported (see Schmucker, 1998, 2005). In regard to mitochondria, it has been reported that aging results in increased mitochondrial size (see Le Couteur et al., 2010)

Exp Gerontol. Author manuscript; available in PMC 2015 August 01. 
and in some cases this increase can be accompanied by modification of cristal morphology (Sastre et al., 1996). These changes have been confirmed in mice with different progeroid syndromes (Cogger et al., 2013; Gregg et al., 2012) strengthening the idea of increased mitochondrial size during aging.

In our study, aging and long-term CR affected hepatocyte mitochondria structure in two ways: by inducing changes in individual mitochondria morphology and in the whole mitochondrial population per hepatocyte. First, we detected changes in planimetric parameters (area, major and minor diameters) which consequently affected mitochondrial volumes. In particular, $\mathrm{CR}$ resulted in increased mitochondrial number and volume. However, these results also depended on the fat source since significant differences were found when comparing the CR groups. In this case, CR-Lard and CR-Soy exhibited larger mitochondria than the CR-Fish group. Also, mitochondrial shape varied with CR in such a way that more spherical mitochondria were found in all the CR groups regardless of dietary fat. Finally, we found substantial increases in both mean number of cristae per mitochondria and mean crista length when comparing CR animals with controls, but once again differences were found when comparing CR-groups consuming diets differing in lipid composition. In this case, a sequential increase in cristal length was found with CR-Soy > CR-Lard > CR-Fish > control. These results contrast with those reported in young mice subjected to 6 month of CR (Khraiwesh et al., 2013) where increased mitochondria were found only in the CR-Lard group (see Table 6). Thus, mitochondrial response to CR and dietary lipid composition is influenced by age and/or duration of CR.

Mean number of mitochondrial cristae and crista length increased in all CR mice compared to AL animals, a phenomenon that we had found in young animals following 6 months of CR (Khraiwesh et al., 2013). Since mitochondrial crista is the location of the electron transport chain and oxidative phosphorylation, the possibility exists that the increase in crista surface could be part of the metabolic adaptation to the low energy status imposed by CR. In a previous study focused on young animals fed with the same experimental diets for 1 month we reported that CR decreased ROS production by complex III but did not markedly alter proton leak and electron transport chain enzyme activities. Interestingly, these mitochondrial functions were influenced by dietary lipid composition in CR animals (Chen et al., 2013). The structural changes of hepatocyte mitochondria by CR and dietary fat that we report here could be related with these modifications of mitochondrial physiology.

\subsection{Mitochondrial mass and number of mitochondria per hepatocyte}

Taken as a whole, the mitochondrial compartment, i.e. the set of mitochondria contained in a hepatocyte, appears to undergo remarkable changes induced by aging and dietary interventions, including CR. In some cases these changes appear after a few weeks of the intervention, denoting a quick adaptation of the mitochondria compartment to the conditions induced by these interventions. In spite of some contradictory data (see Pieri et al., 1980), it is assumed that aging results in a decreased number of mitochondria in hepatocytes (Sastre et al., 1996; Schmucker et al., 1978), an effect that has also been observed in progeroid Werner mice (Cogger et al., 2013). Conversely, CR has been reported to induce a significant increase in the number of mitochondria per cell (López-Lluch et al., 2006). Resveratrol, a 
partial CR mimetic, had the same effect in middle-aged mice fed with a high diet (Baur et al., 2006) as well as in senescence-accelerated mice (Shiozaki et al., 2011). Consistent with these results, we found significant increases in mitochondrial mass and in the mean number of mitochondria per hepatocyte in all CR groups regardless of dietary fat when compared with AL controls. However, the extent of these increases in mitochondrial number differed between CR groups with the CR-Soy and CR-Fish groups showing greater mitochondrial mass than the CR-Lard group.

When comparing the results included here with those reported in our previous work carried out on mice subjected to 6 months of CR (Khraiwesh et al., 2013), we found that both mitochondrial mass and number decreased by $20 \%$ in the old versus young control (AL) fed animals, which is in accordance with the results described above. Also, when comparing the $\mathrm{CR}$ groups between studies some differences arose. In the CR-Soy group the number of mitochondria per hepatocyte remained fairly constant with age while a decrease (about 18\%) was found in the CR-Lard group and an increase of $30 \%$ was observed in the CR-Fish animals (Table 6). These results indicate that the dietary fat plays a role in the morphological accommodation of mitochondrial compartment to the temporal and physiological conditions imposed by CR.

\subsection{Mitochondrial dynamics markers, aging and calorie restriction}

Mitochondrial biogenesis and metabolism are a complex and highly regulated process operating through PGC-1a dependent nuclear respiratory factors such as Nrf1 (López-Lluch et al., 2006). Although some studies have showed increased expression of mRNA of PGC-1a and Nrf1 and protein (Nisoli et al., 2005) following CR, others have found no effect of CR in these markers of mitochondrial biogenesis. In our study we have detected increases of both factors in old animals from AL group. However, under CR conditions increased levels of PGC-1a were only found in the CR-Lard group following 18 months of CR. Similarly, expression levels of Nrf1 increased in AL old animals. Nevertheless, longterm calorie restriction resulted in increased Nrf1 levels in both CR-Lard and CR-Fish groups.

Mitochondria are highly dynamic organelles that undergo continuous fission and fusion processes. Fis1 and Drp1 mediate mitochondrial fission, while Mfn1, Mfn2 and OPA1 are implicated in fusion. The equilibrium between both phenomena defines mitochondrial ultrastructure and abundance in the different cell types and under the diverse physiological conditions (see Chan, 2012 for a recent review). In our experiments we have found relatively stable expression levels of fission and fusion proteins when comparing AL with CR groups. However, Fis1 expression levels decreased in CR-Soy versus AL while no changes related to dietary fat were found in the CR groups. With regard to the mitochondrial fusion-related proteins, we detected decreased levels of OPA1 expression in the CR groups containing Lard or Fish as dietary fat when compared to the CR-Soy group, but no differences were found when comparing AL controls with the CR-Soy group.

In hepatocytes, calorie restriction has been reported to increase mitochondrial biogenesis, and the same result was obtained in high calorie fed animals supplemented with resveratrol compared to their corresponding controls (Baur et al., 2006; López-Lluch et al., 2006). 
Although we have found a substantial increase in mitochondrial mass after 18 months of CR with different dietary fats, no differences in the levels of protein expression related to mitochondrial fission were detected. Furthermore, a decrease in Fis1 expression was found in the CR-Soy group. In a similar way, we did not find striking changes in the expression level of proteins related to mitochondrial fusion except for OPA1 which significantly increased in the CR-Soy compared to the other CR groups. In addition to its role in mitochondrial internal membrane fusion,OPA1 plays an important role in morphology and remodeling crista structure (see Chan, 2012) and interestingly, CR-Soy fed animals showed the highest number and longest cristae compared to all other groups. This is in accordance with the results of Gomes et al. (2011) who reported increased mitochondrial size and crista number in different cell types upon starvation.

In general, the expression levels of proteins related to mitochondrial fission and fusion as well as PGC-1a and Nrf1 were higher or remained unaltered when compared with the corresponding young animal counterparts after 6 months of CR (Khraiwesh et al., 2013). This result is compatible with CR opposing age-related decreases in mitochondrial biogenesis. Furthermore, the studies highlight dietary fat as a crucial factor in the regulation of mitochondria structure and abundance in hepatocytes under CR conditions.

\subsection{Hydroperoxide levels, aging and calorie restriction}

The results included in this paper show no age-related changes of hydroperoxide levels in AL group. However, CR induced several changes in this parameter. Thus, in animals fed for 6 months under CR we found a sequential increase of hydroperoxide levels in the three dietary groups ordered as Lard-Soy-Fish, these levels being significantly higher in CR-Fish when compared to CR-Lard. However, after 18 months of CR we found increased values only in CR-Lard group, remaining unaltered in AL and CR-Soy groups. Interestingly, a decrease of hydroperoxide levels was found in old animals from CR-Fish group. Recently, we have reported that the transgenic mice fat-1, which are capable to synthesizing n-3 fatty acids, decrease the rate of mitochondrial hydrogen peroxide generation, providing a defense mechanism against oxidative stress by limiting ROS production (Hagopian etal., 2010). Thus, the possibility exists that under calorie restriction, animal fed diets enriched in n-3 fatty acids which are prone to oxidative damage (such as those from CR-Fish group), could develop a similar defense mechanism by reducing ROS production without additional hydroperoxide accumulation after 18 months of CR. These results indicate a possible mechanism of adaptation of animals to diets containing a high proportion of fatty acid susceptible to peroxidation and oxidative stress.

A relationship between mitochondrial dynamics and cell adaptation to nutrient availability has been suggested, such that different mechanisms may operate in nutrient excess or under starvation in order to achieve a more efficient mitochondrial population (Liesa and Shirihai, 2013). In our case, long-term CR induces differential increases in the hepatocyte mitochondrial population and the possibility exists that mitochondrial fission/fusion machinery could be subjected to different control mechanisms depending on the dietary fat. Alterations in fatty acid composition of mitochondrial phospholipids induced by the experimental diets (Chen et al., 2012, 2013) could also play a role in the metabolic 
adaptation of this organelle. This possibility needs to be further investigated in future studies.

\section{Conclusions}

The results included in this paper show significant changes in both hepatocyte size and mitochondrial ultrastructure in hepatocytes from mature/old mice following long-term calorie restriction. Furthermore, we have found significant changes in mitochondrial biogenesis and metabolism regulators PGC-1a and Nrf1 as well as in protein related to mitochondrial fission and fusion. The extent of these changes depended on dietary fat composition. Thus, the results of this study indicate that dietary fat composition plays a crucial role in the fine tuning of the mitochondria and hepatocyte relationship. Furthermore, dietary fat composition should be taken into consideration when designing diets to optimize the mitochondrial changes induced by $\mathrm{CR}$.

\section{Acknowledgments}

Supported by NIH grant 1R01AG028125-01A1 (to JJR, PN and JMV), Ministerio de Economía y Competitividad and European FEDER BFU2011-23578 (to JMV), Junta de Andalucía Proyectos de Excelencia grant P09CVI-4887 (to JMV), Junta de Andalucía Proyectos Internacionales (to JMV), and BIO-276 (Junta de Andalucía and the University of Córdoba, to JMV and EGC). JALD and LFdR were funded by predoctoral fellowships of the Spanish Ministerio de Educación and by BIO-276. HK was funded by a predoctoral fellowship of the Agencia Española de Cooperación Internacional al Desarrollo and by BIO-276.

\section{References}

Barja G. Updating the mitochondrial free radical theory of aging: an integrated view, key aspects, and confounding concepts. Antioxid. Redox Signal. 2013; 19:1420-1445. [PubMed: 23642158]

Baur JA, Pearson KJ, Price NL, Jamieson HA, Lerin C, Kalra A, Prabhu VV, Allard JS, Lopez-Lluch G, Lewis K, Pistell PJ, Poosala S, Becker KG, Boss O, Gwinn D, Wang M, Ramaswamy S, Fishbein KW, Spencer RG, Lakatta EG, Le Couteur D, Shaw RJ, Navas P, Puigserver P, Ingram DK, de Cabo R, Sinclair DA. Resveratrol improves health and survival of mice on a high-calorie diet. Nature. 2006; 444:337-342. [PubMed: 17086191]

Bello RI, Alcaín FJ, Gómez-Díaz C, López-Lluch G, Navas P, Villalba JM. Hydrogen peroxide- and cell-density-regulated expression of NADH-cytochrome b5 reductase in HeLa cells. J. Bioenerg. Biomembr. 2003; 35:169-179. [PubMed: 12887015]

Bevilacqua L, Ramsey JJ, Hagopian K, Weindruch R, Harper ME. Effects of short- and medium-term calorie restriction on muscle mitochondrial proton leak and reactive oxygen species production. Am. J. Physiol. Endocrinol. Metab. 2004; 286:E852-E861. [PubMed: 14736705]

Bratic A, Larsson NG. The role of mitochondria in aging. J. Clin. Invest. 2013; 123:951-957. [PubMed: 23454757]

Campisi J. Aging, cellular senescence, and cancer. Annu. Rev. Physiol. 2013; 75:685-705. [PubMed: 23140366]

Chan DC. Fusion and fission: interlinked processes critical for mitochondrial health. Annu. Rev. Genet. 2012; 46:265-287. [PubMed: 22934639]

Chen Y, Hagopian K, McDonald RB, Bibus D, López-Lluch G, Villalba JM, Navas P, Ramsey JJ. The influence of dietary lipid composition on skeletal muscle mitochondria from mice following 1 month of calorie restriction. J. Gerontol. A Biol. Sci. Med. Sci. 2012; 67:1121-1131. [PubMed: 22503990]

Chen Y, Hagopian K, Bibus D, Villalba JM, López-Lluch G, Navas P, Kim K, McDonald RB, Ramsey JJ. The influence of dietary lipid composition on liver mitochondria from mice following 1 month of calorie restriction. Biosci. Rep. 2013; 33:83-95. [PubMed: 23098316] 
Chung KW, Kima DH, Parka MH, Choia YJ, Kima ND, Leea J, Yub BP, Chung HY. Recent advances in calorie restriction research on aging. Exp. Gerontol. 2013; 48:1049-1053. [PubMed: 23201549]

Cogger VC, Svistounov D, Warren A, Zykova S, Melvin RG, Solon-Biet SM, O'Reilly JN, McMahon AC, Ballard JW, De Cabo R, Le Couteur DG, Lebel M. Liver aging and pseudocapillarization in a Werner syndrome mouse model. J. Gerontol. A Biol.Sci. Med. Sci. 2013 http://dx.doi.org/10.1093/ gerona/glt169 (Oct 22, Epub ahead of print).

Colman RJ, Anderson RM, Johnson SC, Kastman EK, Kosmatka KJ, Beasley TM, Allison DB, Cruzen C, Simmons HA, Kemnitz JW, Weindruch R. Caloric restriction delays disease onset and mortality in rhesus monkeys. Science. 2009; 325:201-204. [PubMed: 19590001]

Gomes LC, Di Benedetto G, Scorrano L. During autophagy mitochondria elongate, are spared from degradation and sustain cell viability. Nat. Cell Biol. 2011; 13:589-598. [PubMed: 21478857]

Gregg SQ, Gutiérrez V, Robinson AR, Woodell T, Nakao A, Ross MA, Michalopoulos GK, Rigatti L, Rothermel CE, Kamileri I, Garinis GA, Stolz DB, Niedernhofer LJ. A mouse model of accelerated liver aging caused by a defect in DNA repair. Hepatology. 2012; 55:609-621. [PubMed: 21953681]

Hagopian K, Harper ME, Ram JJ, Humble SJ, Weindruch R, Ramsey JJ. Long-term calorie restriction reduces proton leak and hydrogen peroxide production in liver mitochondria. Am. J. Physiol. Endocrinol. Metab. 2005; 288:E674-E684. [PubMed: 15562252]

Hagopian K, Weber KL, Hwee DT, Van Eenennaam AL, López-Lluch G, Villalva JM, Burón I, Navas P, German JB, Watkins SM, Chen Y, Wei A, McDonald RB, Ramsey JJ. Complex I-associated hydrogen peroxide production is decreased and electron transport chain enzyme activities are altered in n-3 enriched fat-1 Mice. PLoS ONE. 2010; 5(9):e12696. http://dx.doi.org/10.1371/ journal.pone.0012696. [PubMed: 20856881]

Harman D. The biological clock: the mitochondria? J. Am. Geriatr. Soc. 1972; 20:145-147. [PubMed: 5016631]

Hekimi S, Lapointe J, Wen Y. Taking a "good" look at free radicals in the aging process. Trends Cell Biol. 2011; 21:569-576. [PubMed: 21824781]

Hulbert AJ. Life, death and membrane bilayers. J. Exp. Biol. 2003; 206:2303-2311. [PubMed: 12796449]

Jiang ZY, Woollard AC, Wolff SP. Lipid hydroperoxide measurement by oxidation of $\mathrm{Fe} 2+$ in the presence of xylenol orange. Comparison with the TBA assay and an iodometric method. Lipids. 1991; 26:853-856. [PubMed: 1795606]

Kaneko T, Tahara S, Matsuo M. Retarding effect of dietary restriction on the accumulation of 8hydroxy-2'-deoxyguanosine in organs of Fischer 344 rats during aging. Free Radic Biol. Med. 1997; 23:76-81. [PubMed: 9165299]

Khraiwesh H, López-Domínguez JA, López-Lluch G, Navas P, de Cabo R, Ramsey JJ, Villalba JM, González-Reyes JA. Alterations of ultrastructural and fission/fusion markers in hepatocyte mitochondria from mice following calorie restriction with different dietary fats. J.Gerontol. A Biol. Sci. Med. Sci. 2013; 68:1023-1034. [PubMed: 23403066]

Le Couteur, DG.; Sinclair, DA.; Cogger, VC. The aging liver and the effect of longterm caloric restriction. In: Everitt, AV.; Rattan, SIS.; Le Couteur, DG.; de Cabo, R., editors. Calorie Restriction, Aging and Longevity. Dordrecht, Netherlands: Springer Press; 2010. p. 191-216.

Liesa M, Shirihai OS. Mitochondrial dynamics in the regulation of nutrient utilization and energy expenditure. Cell Metab. 2013; 17:491-506. [PubMed: 23562075]

López-Domínguez JA, Khraiwesh H, González-Reyes JA, López-Lluch G, Navas P, Ramsey JJ, de Cabo R, Burón MI, Villalba JM. Dietary fat modifies mitochondrial and plasma membrane apoptotic signaling in skeletal muscle of calorie restricted mice. Age. 2013; 35:2027-2044. [PubMed: 23179253]

López-Domínguez JA, Khraiwesh H, González-Reyes JA, López-Lluch G, Navas P, Ramsey JJ, de Cabo R, Buron I, Villalba JM. Dietary fat and aging modulate apoptotic signaling in liver of calorie-restricted mice. J.Gerontol. A Biol. Sci. Med. Sci. 2014 http://dx.doi.org/10.1093/gerona/ glu045 (in press). 
López-Lluch G, Hunt N, Jones B, Zhu M, Jamieson H, Hilmer S, Cascajo MV, Allard J, Ingram DK, Navas P, de Cabo R. Calorie restriction induces mitochondrial biogenesis and bioenergetic efficiency. Proc. Natl. Acad. Sci. U. S. A. 2006; 103:1768-1773. [PubMed: 16446459]

López-Otín C, Blasco MA, Partridge L, Serrano M, Kroemer G. The hallmarks of aging. Cell. 2013; 153:1194-1217. [PubMed: 23746838]

Mattison JA, Roth GS, Beasley TM, Tilmont EM, Handy AM, Herbert RL, Longo DL, Allison B, Young JE, Bryant M, Barnard D, Ward WF, Qi W, Ingram DK, de Cabo R. Impact of caloric restriction on health and survival in rhesus monkeys from the NIA study. Nature. 2012; 489:318321. [PubMed: 22932268]

Miquel J, Economos AC, Fleming J, Johnson JE Jr. Mitochondrial role in cell aging. Exp. Gerontol. 1980; 15:575-591. [PubMed: 7009178]

Nisoli E, Tonello C, Cardile A, Cozzi V, Bracale R, Tedesco L, Falcone S, Valerio A, Cantoni O, Clementi E, Moncada S, Carruba MO. Calorie restriction promotes mitochondrial biogenesis by inducing the expression of eNOS. Science. 2005; 310:314-317. [PubMed: 16224023]

Pamplona R, Barja G, Portero-Otin M. Membrane fatty acid unsaturation, protection against oxidative stress, and maximum life span: a homeoviscous-longevity adaptation? Ann. N. Y. Acad. Sci. 2002; 959:475-490. [PubMed: 11976221]

Pieri C, Giuli C, Del Moro M, Piantanelli L. Electron-microscopic morphometric analysis of mouse liver. II. Effect of ageing and thymus transplantation in old animals. Mech. Ageing Dev. 1980; 13:275-283. [PubMed: 7421302]

Sastre J, Pallardo FV, Plá R, Pellín A, Juan G, O'Connor JE, Estrela JM, Miquel J, Viña J. Aging of the liver: age-associated mitochondrial damage in intact hepatocytes. Hepatology. 1996; 24:11991205. [PubMed: 8903398]

Sato T. A modified method for lead staining of thin sections. J. Electron Microsc. (Tokyo). 1968; 17:158-159. [PubMed: 4177281]

Schmucker DL. Hepatocyte fine structure during maturation and senescence. J. Electron Microsc. Tech. 1990; 14:106-125. [PubMed: 2406386]

Schmucker DL. Aging and the liver: an update. J. Gerontol. Biol. Sci. 1998; 53A:B315-B320.

Schmucker DL. Age-related changes in liver structure and function: implications for disease? Exp. Gerontol. 2005; 40:650-659. [PubMed: 16102930]

Schmucker DL, Sanchez H. Liver regeneration and aging: a current perspective. Curr. Gerontol. Geriatr. Res. 2011 http://dx.doi.org/10.1155/2011/526379 (Article ID 526379, 8 pages) 2011.

Schmucker DL, Mooney JS, Jones AL. Stereological analysis of hepatic fine structure in the Fischer 344 rat. Influence of sublobular location and animal age. J. Cell Biol. 1978; 78:319-337. [PubMed: 690169]

Shiozaki M, Hayakawa N, Shibata M, Koike M, Uchiyama Y, Gotow T. Closer association of mitochondria with lipid droplets in hepatocytes and activation of Kupffer cells in resveratroltreated senescence-accelerated mice. Histochem. Cell Biol. 2011; 136:475-489. [PubMed: 21818579]

Sohal RS, Weindruch R. Oxidative stress, caloric restriction, and aging. Science. 1996; 273:59-63. [PubMed: 8658196]

Sohal RS, Agarwal S, Candas M, Forster MJ, Lal H. Effect of age and caloric restriction on DNA oxidative damage in different tissues of C57BL/6 mice. Mech. Ageing Dev. 1994; 76:215-224. [PubMed: 7885066]

Weibel, ER. Stereological Methods. Practical Methods for Biological Morphometry. Vol. 1. New York, NY: Academic Press; 1979.

Weindruch R, Sohal RS. Seminars in medicine of the Beth Israel Deaconess Medical Center. Caloric intake and aging. N. Engl. J. Med. 1997; 337:986-994.

Weindruch, RH.; Walford, RL. The Retardation of Aging and Disease by Dietary Restriction. Springfield, IL: Charles C. Thomas; 1988.

Youngman LD, Park JY, Ames BN. Protein oxidation associated with aging is reduced by dietary restriction of protein or calories. Proc. Natl. Acad. Sci. U. S. A. 1992; 89:9112-9116. [PubMed: 1409611] 
Yu BP, Lim BO, Sugano M. Dietary restriction downregulates free radical and lipid peroxide production: plausible mechanism for elongation of life span. J. Nutr. Sci. Vitaminol. 2002; 48:257-264. [PubMed: 12489815]

Zs.-Nagy I. A membrane hypothesis of aging. J. Theor. Biol. 1978; 75:189-195. [PubMed: 745437] 

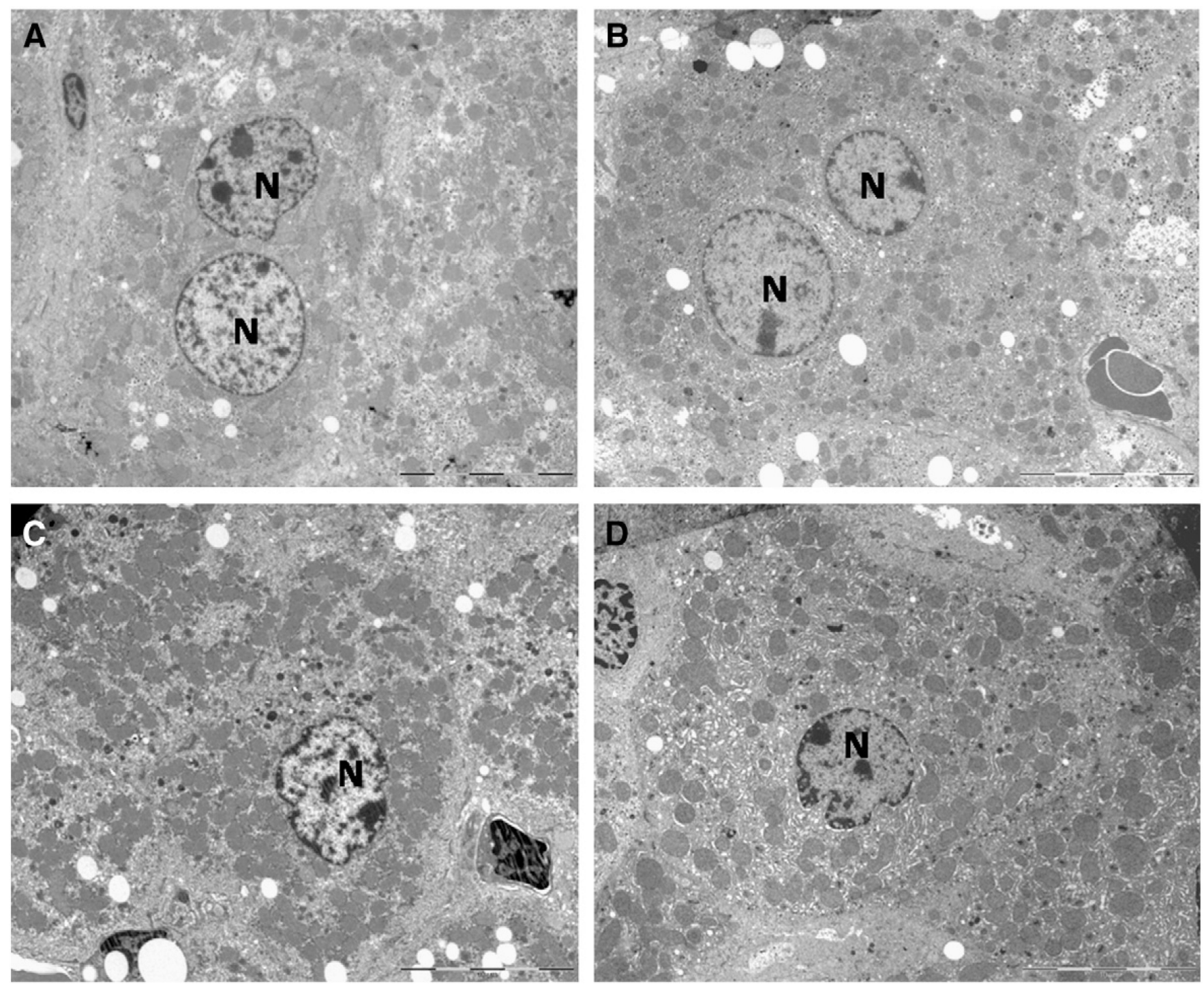

Fig. 1.

Representative pictures of mice hepatocytes following 18 months under the different diets (A, ad libitum; B, CR-Lard; C, CR-Soy and D, CR-Fish) showing the general ultrastructure and preservation of the materials used for the quantitative analysis. Mitochondria were found spread out on the cytoplasm and surrounding the nuclei $(\mathrm{N})$. The bars are equal to 10 $\mu \mathrm{m}$. 

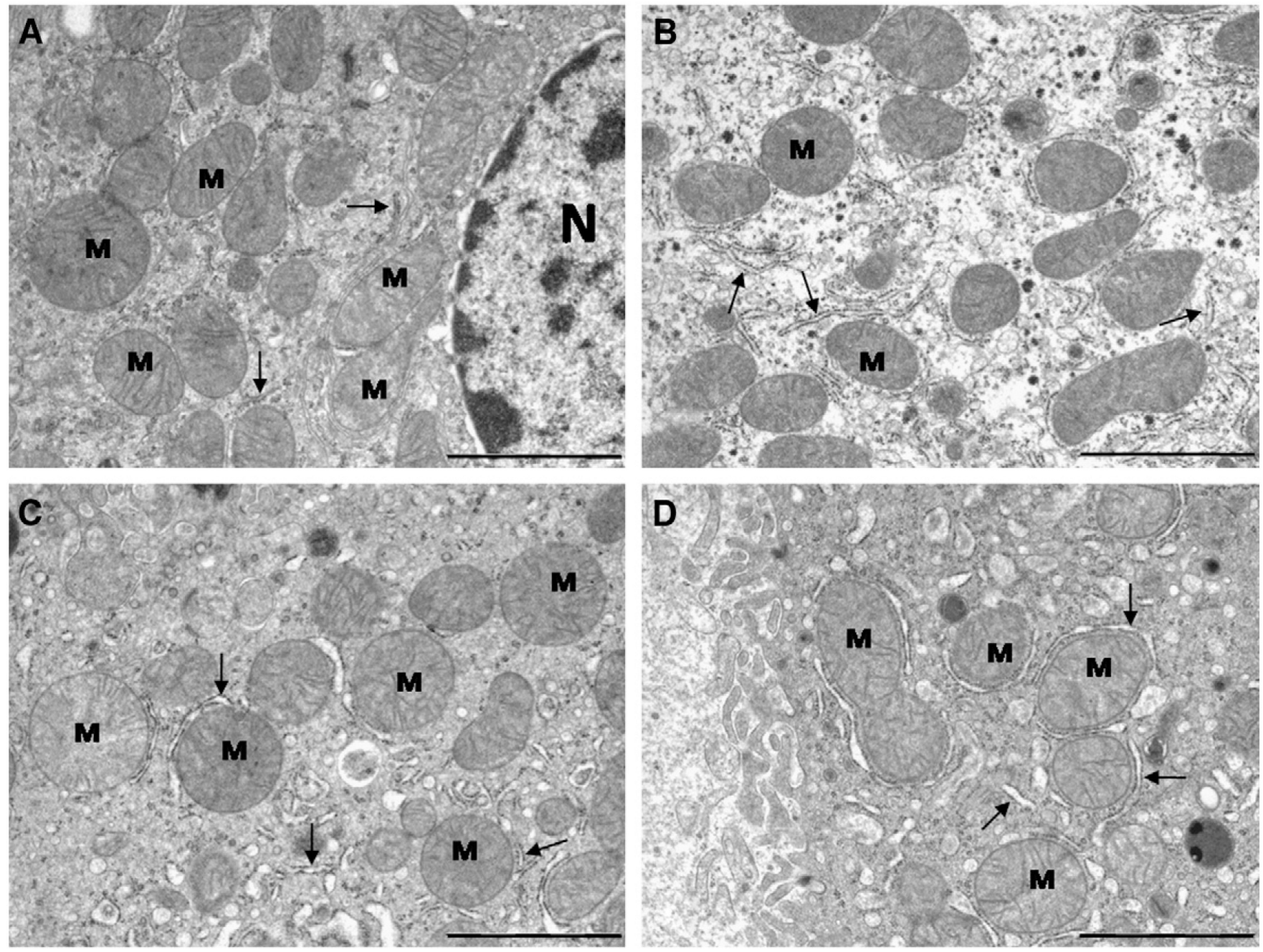

Fig. 2.

Ultrastructural features of mitochondria (M) from mouse hepatocytes fed ad libitum (A)or following 18 month of $40 \%$ CR with different dietary fats (B, CR-Lard; C, CR-Soy and D, CR-Fish). Quantitative changes were detected after planimetric measurements (see text). N, nucleus; arrows, rough endoplasmic reticulum. The bars are equal to $2 \mu \mathrm{m}$. 


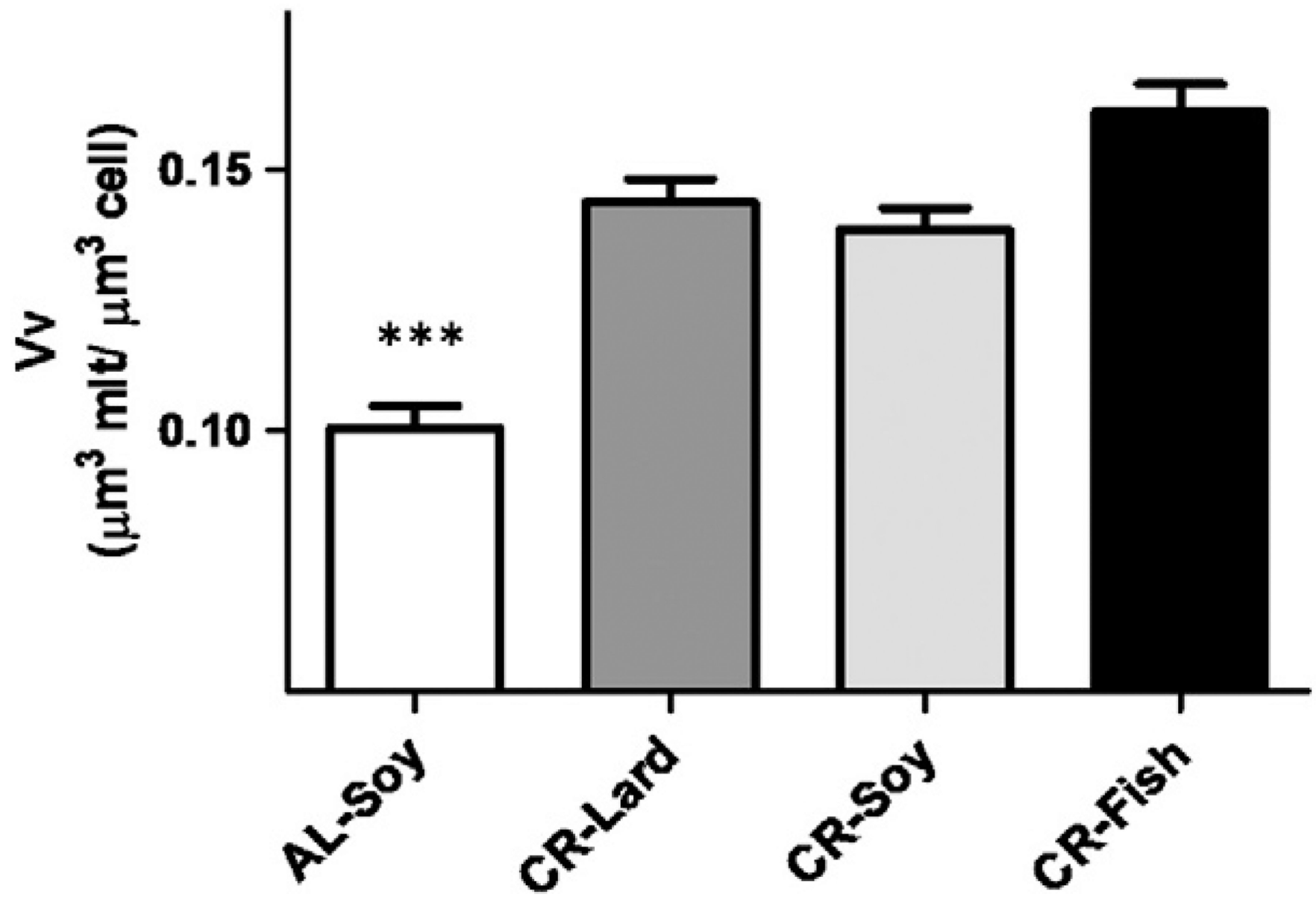

Fig. 3.

Volume density ( $\mathrm{Vv})$ of mitochondria in mouse hepatocytes from the different experimental groups. The cell volume fraction occupied by mitochondria increased under CR regardless the dietary fat compared with ad libitum-fed animals (AL). Data are expressed in $\mu \mathrm{m}^{3}$ of mitochondria per $\mu \mathrm{m}^{3}$ of cell \pm S.E. $* * * \mathrm{p}<.001 \mathrm{vs}$ ad libitum group. 


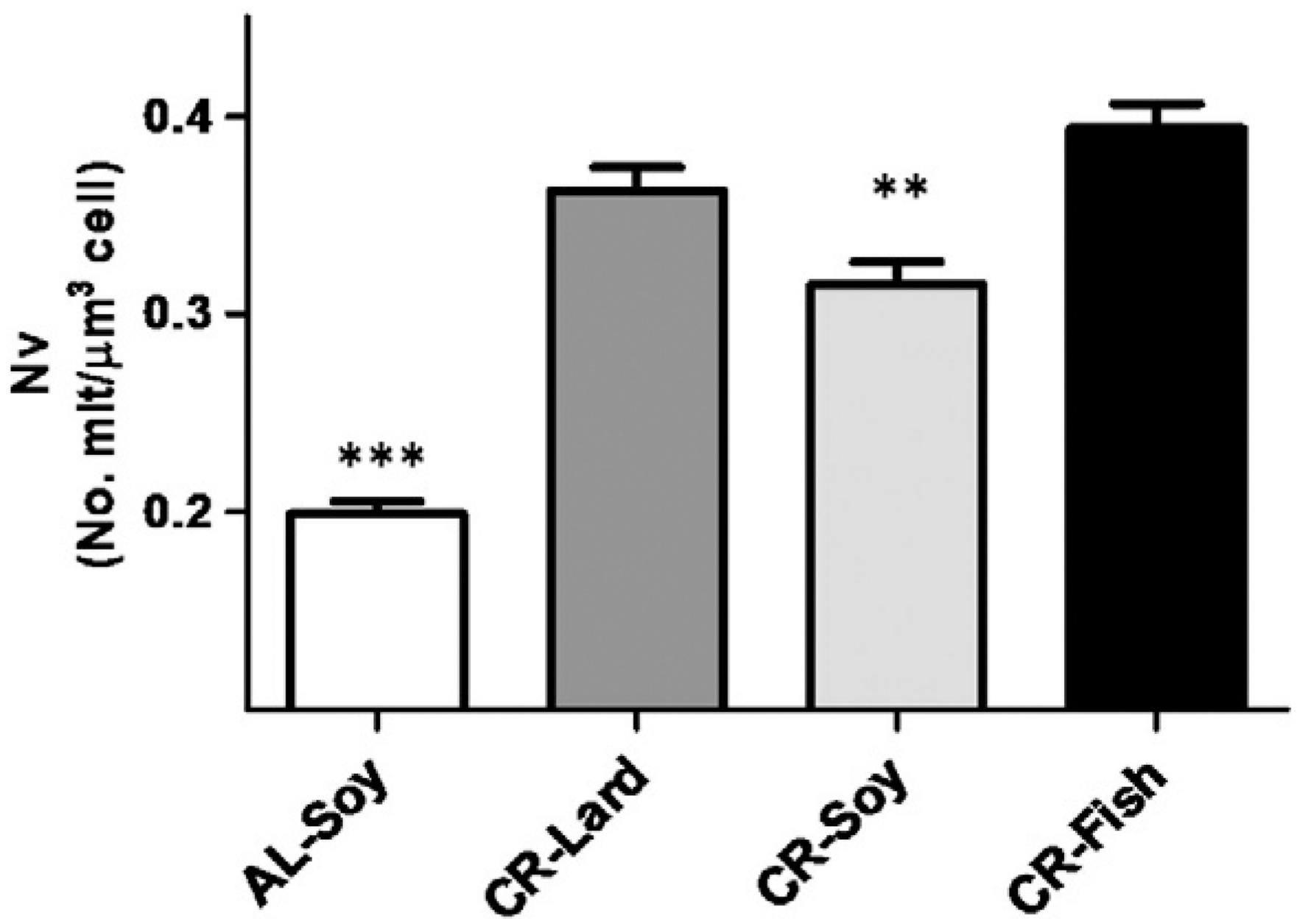

Fig. 4.

Changes in numerical density $(\mathrm{Nv})$ of hepatocyte mitochondria from mice maintained on different diets. This parameter was increased under CR compared with ad libitum-fed animals. Data are expressed as number of mitochondria per $\mu \mathrm{m}^{3}$ of cell \pm S.E. $* * * p<.001$; $* * \mathrm{p}<.01$ vs AL group. 
A
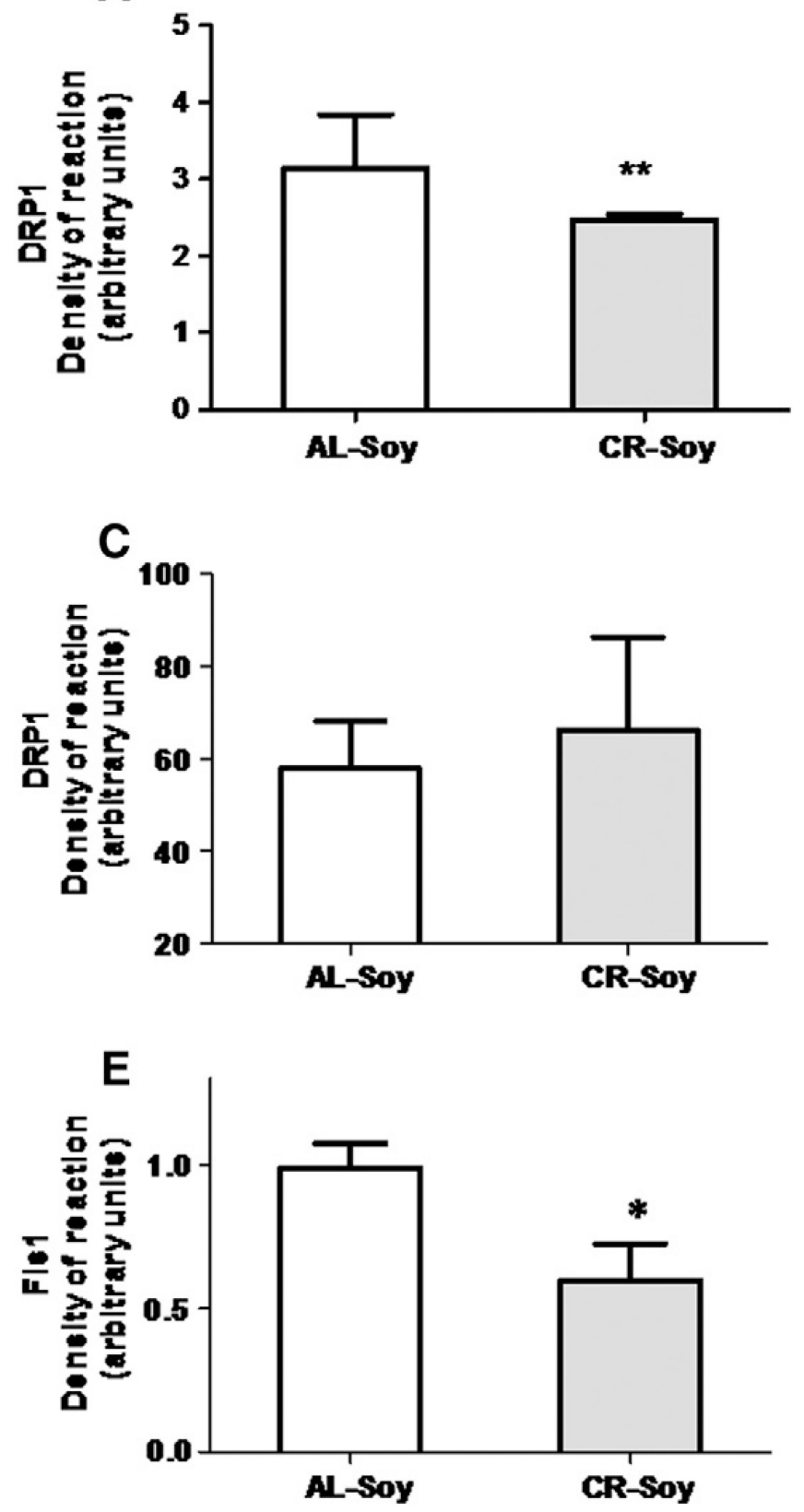

B
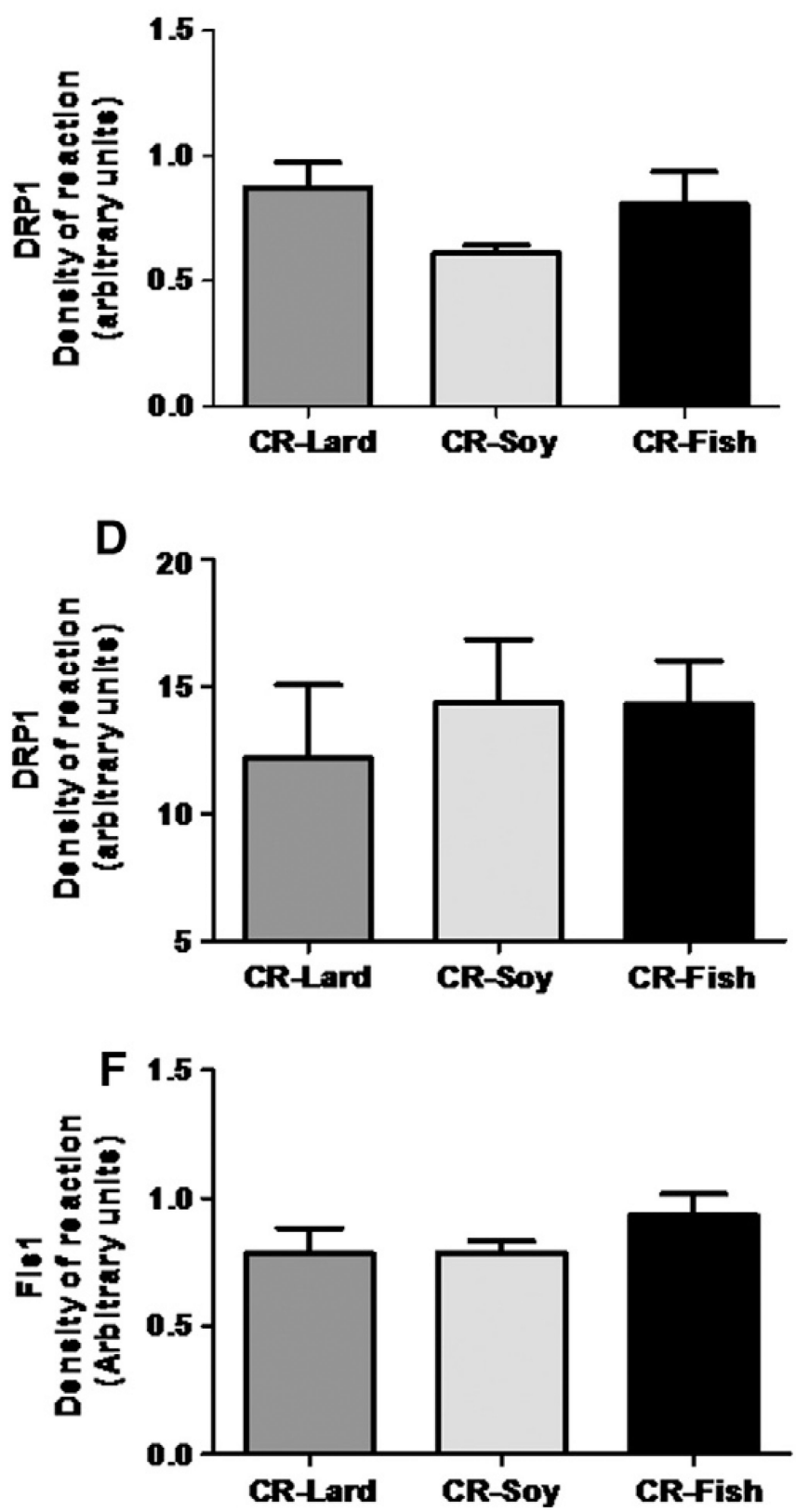

Fig. 5.

Levels of proteins related to mitochondrial fission Drp1 and Fis1. In cytosolic fractions, Drp1 expression decreased $(* * p<.01)$ in calorie restricted animals vs their ad-libitum-fed counterparts (panel A). In this fraction, dietary fat did not induce any additional change in Drp1 (panel B). In mitochondria-enriched fractions, Drp1 protein levels remained unaltered with calorie restriction regardless of the dietary fat (C and D). Fis1 expression level decreased $(* \mathrm{p}<.05)$ under calorie restriction (panel E)but no changes were detected when the different $\mathrm{CR}$ diets were compared among them (panel F). 

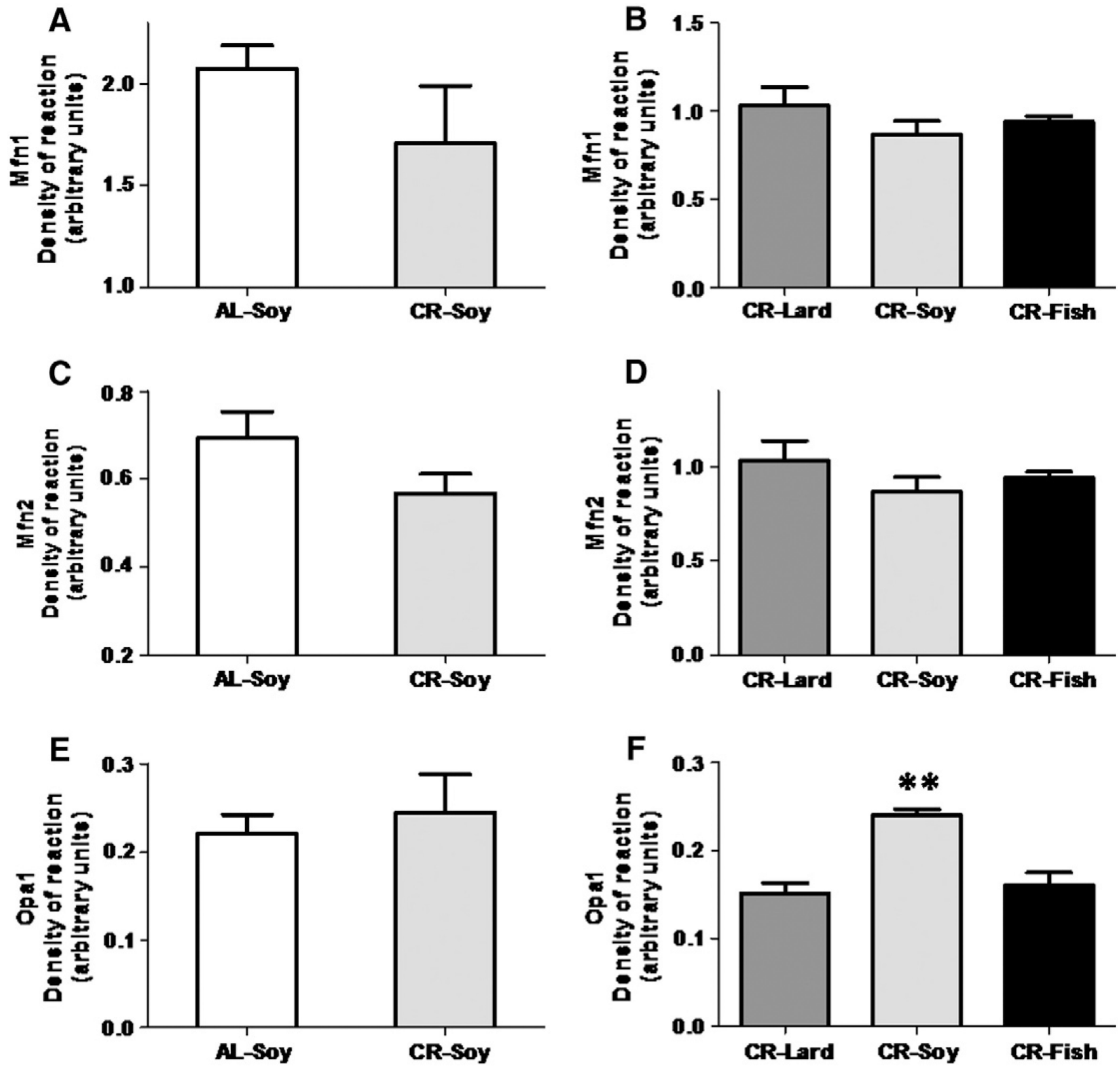

Fig. 6.

Levels of proteins related to mitochondrial fusion: Mfn1 (A and B), Mfn2 (C and D), and OPA1 (E and F). Mfn1 and Mnf2 protein levels remained unaltered either under calorie restriction or due to dietary fat source variations (panels A-D). OPA1 levels remained unchanged when comparing AL versus CR fed animals, but a significant increase was found $(* * \mathrm{p}<.01)$ when comparing CR-Soy with the other dietary fats. 

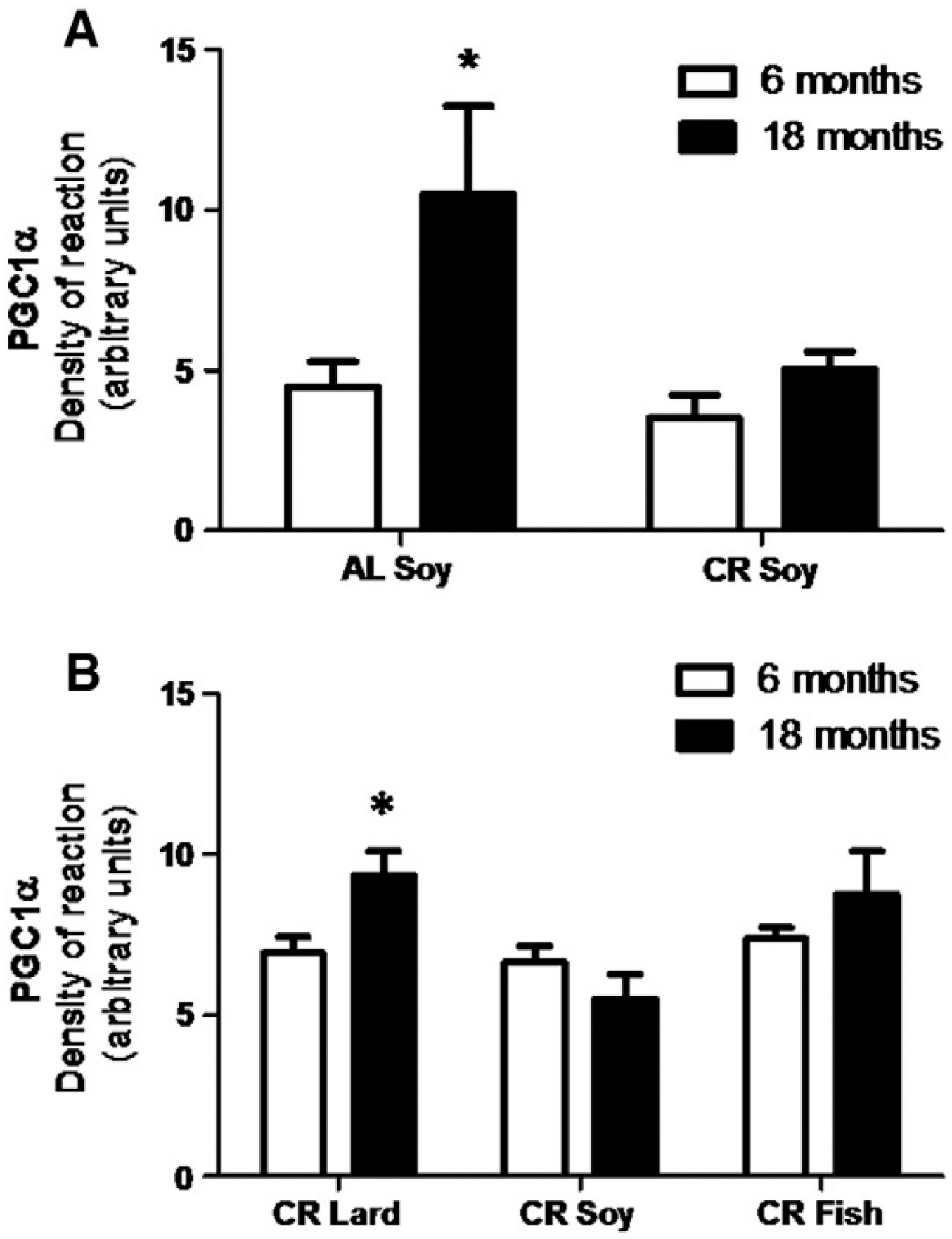

Fig. 7.

Levels of protein expression of PGC-1a measured after 6 and 18 in AL and CR-Soy groups (A) and in the different $\mathrm{CR}$ animals (B). In AL mice an increased value was found in older animals $(* \mathrm{p}<0.05$ vs 6 months). Under calorie restriction age only induced significant increase in CR-Lard group $\left({ }^{*} \mathrm{p}<0.05\right.$ vs 6 months). 

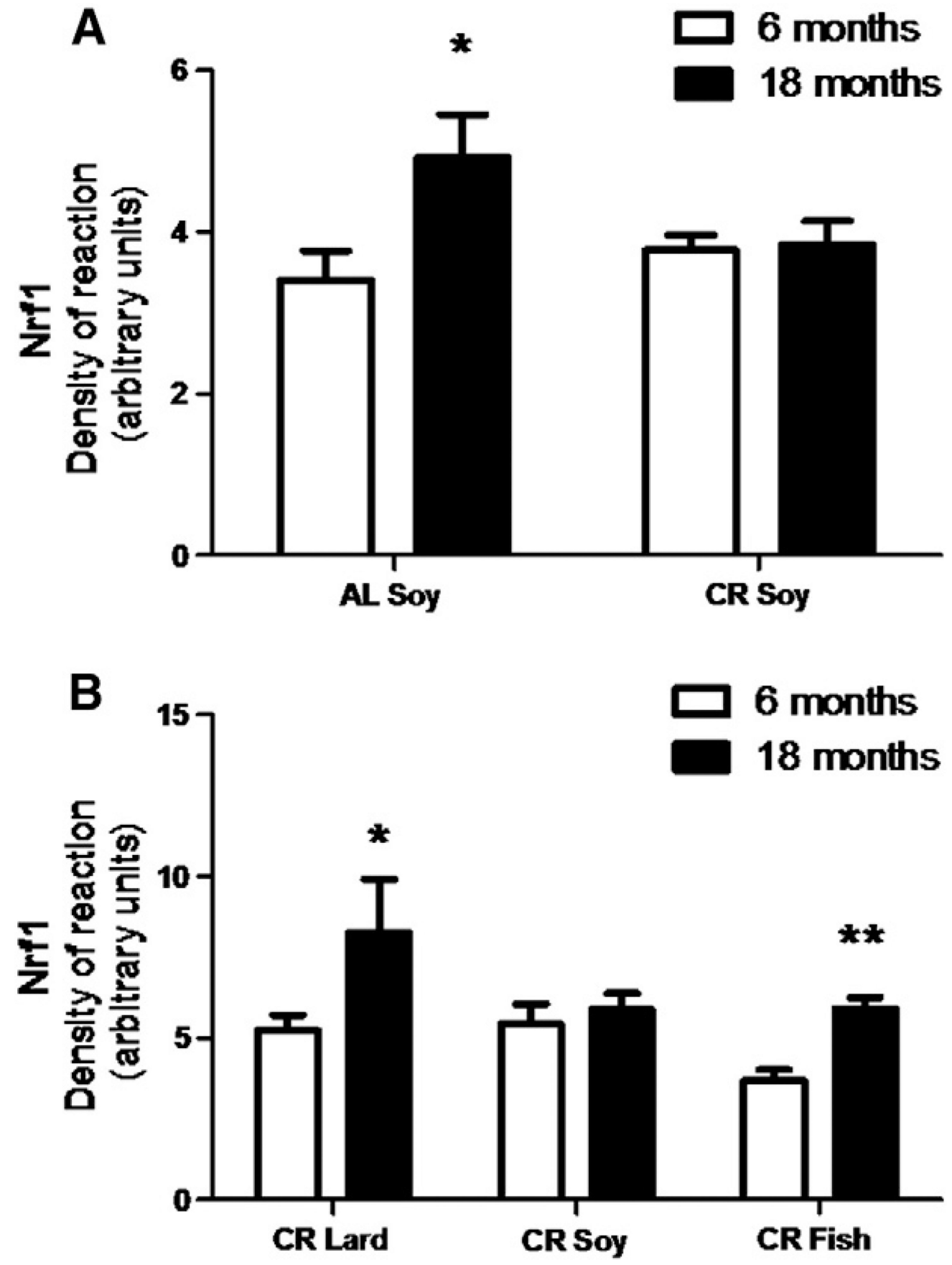

Fig. 8.

Levels of protein expression of Nfr1 measured in mice fed ad libitum (AL) and following 6 and 18 months of calorie restriction with soybean oil as dietary fat (CR-Soy; A). The effects of the different dietary fats under 6 and 18 months of CR are represented in B. In ad libitum fed animals (A), aging induced a significant increase of this parameter ( $p<0.05$ vs 6 months). Under CR (B), both Lard and Fish induced significant increases compared to their respective 6 months of restricted counterparts ( $* \mathrm{p}<0.05$ vs 6 months of CR-Lard; **p < 0.01 vs 6 months of CR-Fish). 
$\square 6$ months

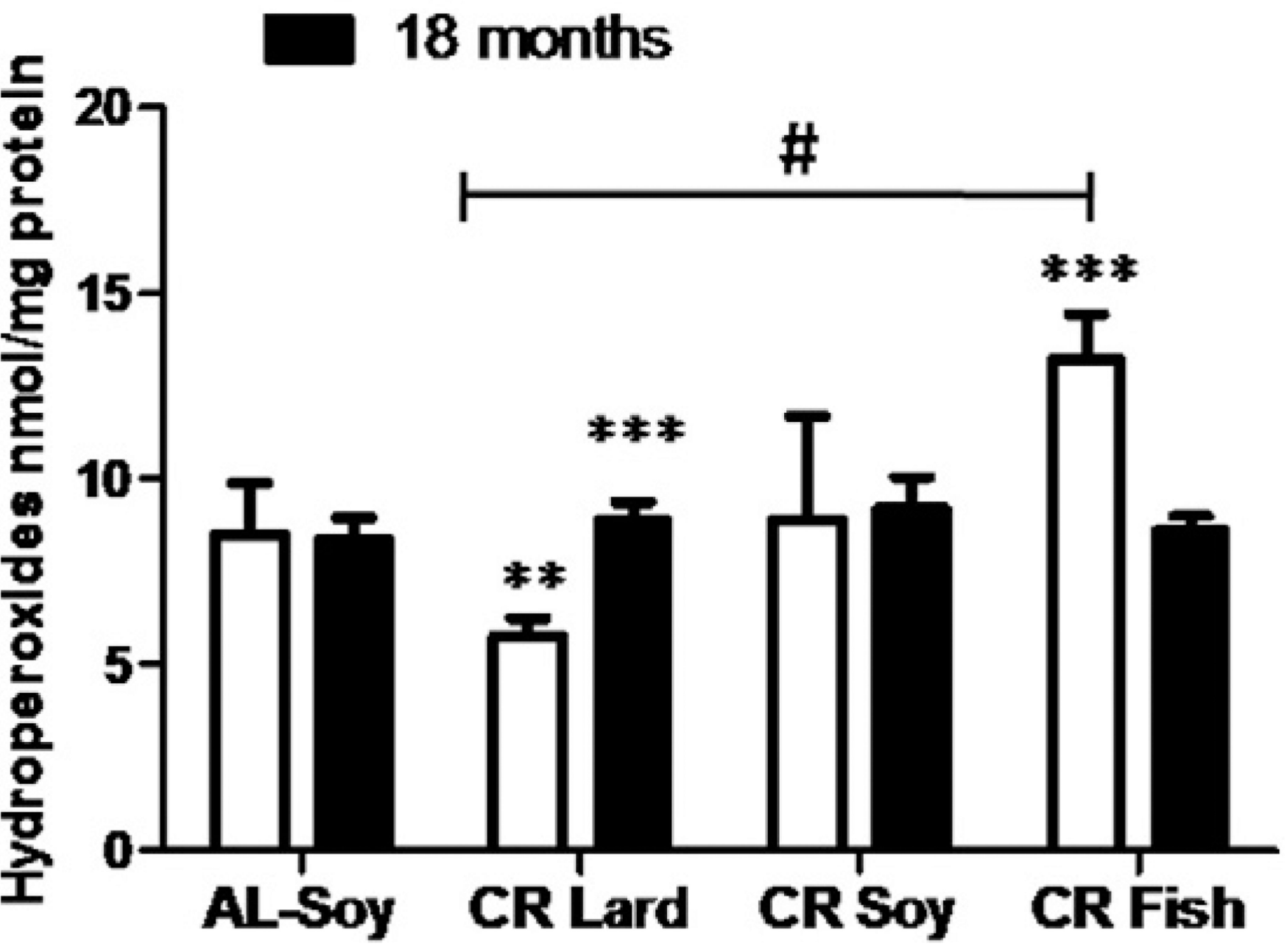

Fig. 9.

Determination of hydroperoxide levels in liver homogenates from mice following 6 or 18 months of calorie restriction with different dietary fats. After 6 months no differences were found when comparing AL controls with the different CR groups. However, when different CR diets were compared, a significant linear trend (\#) was observed, giving CR-Lard dietary group the lowest value and CR-Fish the highest one. Hydroperoxide levels remained unaltered in all the dietary group, but some differences were found when comparing mice fed for 6 or 18 months with CR-Lard and CR-Fish diets $(* * * p<0.001)$. 
Table 1

Main properties of fatty acids present in the three dietary fats.

\begin{tabular}{lrrr}
\hline & Lard & Soybean oil & Fish oil \\
\hline Saturates & $40.3 \%$ & $14.8 \%$ & $28.3 \%$ \\
Monounsaturated fatty acids (18:1 n-9) & $39.2 \%$ & $21.2 \%$ & $8.7 \%$ \\
Total n-6 & $16.0 \%$ & $55.0 \%$ & $3.2 \%$ \\
Total n-3 & $0.7 \%$ & $8.1 \%$ & $33.9 \%$ \\
n-6/n-3 & 24.4 & 6.8 & 0.1 \\
\hline
\end{tabular}




\section{Table 2}

Hepatocyte planimetric parameters measured in semithick sections stained with toluidine blue. Data are mean values \pm S.E.

\begin{tabular}{lllll}
\hline & AL & CR-Lard & CR-Soy & CR-Fish \\
\hline Area $\left(\mu \mathrm{m}^{2}\right)$ & $409.8 \pm 3.8^{a}$ & $358.4 \pm 3.2^{a}$ & $463.2 \pm 4.1^{b}$ & $443.8 \pm 3.9$ \\
Major diameter $(\mu \mathrm{m})$ & $26.5 \pm 0.14^{a}$ & $24.7 \pm 0.12^{a}$ & $28.3 \pm 0.15^{b}$ & $27.6 \pm 0.14$ \\
Minor diameter $(\mu \mathrm{m})$ & $19.4 \pm 0.10^{a}$ & $18.2 \pm 0.09^{a}$ & $20.5 \pm 0.10^{b}$ & $20.1 \pm 0.12$ \\
Circularity & $0.87 \pm 0.0015$ & $0.88 \pm 0.0012^{c}$ & $0.86 \pm 0.0014$ & $0.87 \pm 0.0014$ \\
Cell volume $\left(\mu \mathrm{m}^{3}\right)$ & $5546 \pm 77.8^{a}$ & $4559 \pm 63.6^{a}$ & $6600 \pm 87.3^{b}$ & $6226 \pm 84.8$ \\
$\mathrm{n}$ & 1128 & 1237 & 1126 & 1111 \\
\hline
\end{tabular}

\footnotetext{
$a_{\mathrm{p}}<0.001$ vs all other groups.

$b_{\mathrm{p}}<0.05$ vs CR-Fish.

$c_{\mathrm{p}}<0.01$ vs CR-Soy.
} 
Table 3

Mitochondrial planimetric parameters measured in samples from electron microscopy pictures. Data are mean values \pm S.E.

\begin{tabular}{lllll}
\hline & AL & CR-Lard & CR-Soy & CR-Fish \\
\hline Mean mitochondrial section area $\left(\mu \mathrm{m}^{2}\right)$ & $0.66 \pm 0.012^{a}$ & $0.85 \pm 0.017^{b}$ & $0.89 \pm 0.016^{c}$ & $0.73 \pm 0.015$ \\
Major diameter $(\mu \mathrm{m})$ & $1.03 \pm 0.010^{a}$ & $1.15 \pm 0.012^{c}$ & $1.16 \pm 0.011^{c}$ & $1.06 \pm 0.012$ \\
Minor diameter $(\mu \mathrm{m})$ & $0.79 \pm 0.008^{a}$ & $0.91 \pm 0.011^{d}$ & $0.95 \pm 0.009^{c}$ & $0.85 \pm 0.010$ \\
Circularity & $0.93 \pm 0.004^{a}$ & $0.95 \pm 0.004$ & $0.95 \pm 0.002$ & $0.95 \pm 0.002$ \\
Mean mitochondrial volume $\left(\mu \mathrm{m}^{3}\right)$ & $0.505 \pm 0.014^{a}$ & $0.700 \pm 0.020^{c}$ & $0.740 \pm 0.019^{c}$ & $0.565 \pm 0.018$ \\
$\mathrm{n}$ & 621 & 404 & 510 & 427 \\
\hline
\end{tabular}

\footnotetext{
$a_{\mathrm{p}}<0.001$ vs all other groups.

$b_{\mathrm{p}}<0.05$ vs CR-Soy and $\mathrm{p}<0.001$ vs CR-Fish.

$c_{\mathrm{p}}<0.001$ vs CR-Fish.

$d_{\mathrm{p}}<0.05$ vs CR-Soy and CR-Fish.
} 


\section{Table 4}

Morphometric analysis of mitochondrial cristae in hepatocytes from the different dietary groups (mean values \pm S.E.).

\begin{tabular}{lllll}
\hline & AL & CR-Lard & CR-Soy & CR-Fish \\
\hline Mean number of cristae/mitochondrion & $14.32 \pm 0.225^{a}$ & $20.78 \pm 0.389$ & $22.13 \pm 0.381$ & $20.89 \pm 0.317$ \\
Scored mitochondria $(\mathrm{n})$ & 621 & 431 & 571 & 453 \\
Mean crista length $(\mu \mathrm{m})$ & $0.2399 \pm 0.0010^{a}$ & $0.3113 \pm 0.0013^{b}$ & $0.3338 \pm 0.0013^{c}$ & $0.2851 \pm 0.0012$ \\
Scored cristae $(\mathrm{n})$ & 9218 & 9003 & 12,724 & 9458 \\
\hline
\end{tabular}

$a_{\mathrm{p}}<0.001$ vs all other groups.

$b_{\mathrm{p}}<0.001$ vs CR-Soy and CR-Fish.

$c_{\mathrm{p}}<0.001$ vs CR-Fish. 


\section{Table 5}

Mitochondrial mass (in $\mu \mathrm{m}^{3}$ per cell) and mean number of mitochondria per cell in hepatocytes from mice fed ad libitum (AL) or $40 \%$ calorie restriction (CR) with different dietary fats during 18 months. Data are expressed as mean \pm S.E. The relative change (in \%) in relation to AL-fed animals is indicated within parentheses.

\begin{tabular}{lcccc}
\hline & AL & CR-Lard & CR-Soy & CR-Fish \\
\hline Total mitochondrial mass & $556.3 \pm 30.5^{a}$ & $638.64 \pm 29.6(14 \%)^{a}$ & $901.8 \pm 38.8(62 \%)$ & $1003.0 \pm 47.1(80 \%)$ \\
Number of mitochondria per cell & $1053 \pm 45^{b}$ & $1641 \pm 75(56 \%)^{c}$ & $2075 \pm 104(97 \%)^{c}$ & $2527 \pm 116(132 \%)$ \\
\hline$a_{\mathrm{p}<0.001 \text { vs CR-Soy and CR-Fish. }}$ & & & \\
$b_{\mathrm{p}<0.001 \text { vs all other diets }}$ & & & \\
$c_{\mathrm{p}<0.05 \text { vs CR-Fish. }}$ & & &
\end{tabular}




\section{Table 6}

Age-related changes highlighted by comparing the results obtained in animals subjected to 18 months of CR with different dietary fats (this paper) with those obtained in young animals fed with the same experimental diets for 6 months (Khraiwesh et al., 2013). Upward arrows denote increases, downward arrows indicate decreases and n.s. means no significant change. Percentage of change is also indicated.

\begin{tabular}{|c|c|c|c|c|}
\hline & AL-Soy & CR-Lard & CR-Soy & CR-Fish \\
\hline Hepatocyte volume & $\uparrow 19 \% * * *$ & n.s. & $\uparrow 29^{* * *}$ & $\uparrow 19 \% * * *$ \\
\hline Mitochondrial volume & $\uparrow 24 \% * * *$ & $\uparrow 36 \% \%^{* * *}$ & $\uparrow 100 \%$ *** & $\uparrow 64 \%$ *** \\
\hline Number of cristae per mitochondrion & $\downarrow 16 \% * * *$ & n.s. & n.s. & n.s. \\
\hline Crista length & $\uparrow 11 \%{ }^{* * *}$ & $\uparrow 48 \%{ }^{* * *}$ & $\uparrow 57 \%$ *** & $\uparrow 28 \%$ *** \\
\hline Mitochondrial mass & $\downarrow 15 \% *$ & $\downarrow .34 \% *$ & $\downarrow 12 \% *$ & n.s. \\
\hline Number of mitochondria per hepatocyte & $\downarrow 21 \%$ *** & n.s. & n.s. & $\uparrow 29 \%$ *** \\
\hline Drp1 & $\uparrow 70 \%{ }^{* *}$ & $\uparrow 60 \%$ ** & n.s. & $\uparrow 60 \%$ * \\
\hline Fis1 & $\uparrow 40 \%{ }^{*}$ & n.s. & n.s. & n.s. \\
\hline Mfn1 & $\uparrow 150 \%$ ** & n.s. & n.s. & $\uparrow 250 \%$ ** \\
\hline Mfn2 & n.s. & n.s. & n.s. & n.s. \\
\hline OPA1 & $\uparrow 50 \%{ }^{*}$ & n.s. & $\uparrow 45 \%{ }^{*}$ & n.s. \\
\hline \multicolumn{5}{|l|}{ p $<<0.05}$. \\
\hline \multicolumn{5}{|l|}{$* *$ p $<0.01}$. \\
\hline$* * * \quad p<0.001$. & & & & \\
\hline
\end{tabular}

